\title{
ON THE BEZDEK-PACH CONJECTURE FOR CENTRALLY SYMMETRIC CONVEX BODIES
}

\author{
ZSOLT LÁNGI* AND MÁRTON NASZÓDI**
}

\begin{abstract}
The Bezdek-Pach Conjecture asserts that the maximum number of pairwise touching positive homothetic copies of a convex body in $\mathbb{R}^{d}$ is $2^{d}$. It was proved in [7] that the quantity in question is not larger than $2^{d+1}$. We present an improvement to this result by proving the upper bound $3 \cdot 2^{d-1}$ for centrally symmetric bodies. In [1], the one-sided Hadwiger number of a convex body was introduced. We extend this definition, prove an upper bound on the resulting quantity and show a connection with the problem of touching homothetic bodies.
\end{abstract}

\section{INTRODUCTION}

In this paper, $\mathbb{R}^{d}$ denotes the $d$-dimensional Euclidean space, $o$ is its origin and $\mathbb{S}^{d-1}$ is the unit sphere centered at $o$. A convex body $K$ in $\mathbb{R}^{d}$ is a compact, convex set with non-empty interior. A positive homothetic copy of $K$ is a set of the form $\lambda K+t$ where $\lambda>0$ and $t \in \mathbb{R}^{d}$. Two convex sets in $\mathbb{R}^{d}$ are non-overlapping if their interiors are disjoint. Two non-overlapping convex sets with intersecting closures touch each other.

In 1971 , C. M. Petty [8] proved that the maximum cardinality of a family of pairwise touching translates of a convex body $K$ is at most $2^{d}$ with equality if, and only if, $K$ is an affine image of a cube. As an extension of this problem, $\mathrm{K}$. Bezdek and J. Pach [2] conjectured in 1988 that the maximum number of pairwise touching positive homothetic copies of a convex body $K$ in $\mathbb{R}^{d}$ is $2^{d}$. They showed that any such family of homothetic copies has at most $3^{d}$ elements, and if $C$ is a $d$-dimensional Euclidean ball, then the maximum is equal to $d+2$. In 2006, M. Naszódi [7] improved the first estimate of Bezdek and Pach by proving the upper bound $2^{d+1}$.

Our main goal is to investigate this problem for o-symmetric convex bodies; that is, for bodies $K$ satisfying $K=-K$. First, we introduce some notions.

Definition 1. We call a set $S \subset \mathbb{R}^{d}$ symmetrically antipodal if, for any distinct points $p, q \in S$, there is a hyperplane $H$ such that $p \in H, q \in-H$, and both $H$ and $-H$ support conv $S$.

1991 Mathematics Subject Classification. 52C17, 51N20, 51K05, 52A21, 52A37.

Key words and phrases. Bezdek-Pach Conjecture, homothets, packing, Hadwiger number, antipodality.

* Partially supported by the Alberta Ingenuity Fund.

** Partially supported by a Post-doctoral fellowship of the Pacific Institute for the Mathematical Sciences. 
Definition 2. Let $K \in \mathbb{R}^{d}$ be an o-symmetric convex body and $S \subset K$. If the points of $S$ are at pairwise distances 2 in the normed space with unit ball $K$, we say that $S$ is a diametral subset of $K$.

In [6], H. Hadwiger formulated the problem of determining the maximum number of pairwise non-overlapping translates of a convex body $K \subset \mathbb{R}^{d}$ touching $K$. This number is known as the Hadwiger number $H(K)$ of $K$. L. Fejes-Tóth [5] extended this concept to the notion of generalized Hadwiger numbers (cf. also [3]). Another variant is the one-sided Hadwiger number, defined in [1]. We combine the definitions of [5] and [1] in the following manner.

Definition 3. Let $K \subset \mathbb{R}^{d}$ be an o-symmetric convex body and $\alpha>0$. The open (resp. closed) one-sided generalized Hadwiger number $H_{\alpha}^{+}(K)\left(\operatorname{resp} . \bar{H}_{\alpha}^{+}(K)\right)$ of $K$ is the maximum number of pairwise non-overlapping translates of $\alpha K$ that touch $K$ and whose translation vectors are in an open (resp. closed) half-space whose boundary contains the origin. Furthermore, $H_{\infty}^{+}(K)\left(\right.$ resp. $\left.\bar{H}_{\infty}^{+}(K)\right)$ is the maximum number of pairwise non-overlapping translates of $K$ that contain the origin and whose translation vectors are in an open (resp. closed) half-space whose boundary contains the origin.

Our results are summarized in Theorems 1,2 and 3. Theorem 3 is an immediate consequence of Theorems 1 and 2 .

Theorem 1. The following statements are equivalent.

(1) There is an o-symmetric convex body $K \subset \mathbb{R}^{d}$ which has $n$ pairwise touching homothets.

(2) There is a symmetrically antipodal set $S \subset \mathbb{R}^{d+1}$ of $n$ points such that $o \notin \operatorname{conv} S$.

(3) There is an o-symmetric convex body $K \subset \mathbb{R}^{d+1}$ which contains a diametral set $S$ of $n$ points such that $o \notin \operatorname{conv} S$.

(4) There is an o-symmetric convex body $B \subset \mathbb{R}^{d+1}$ with $H_{\infty}^{+}(B) \geq n$.

Theorem 2. Let $\alpha \in(0, \infty), d \geq 2$, and $K$ be an o-symmetric convex body in $\mathbb{R}^{d}$. Then

$$
\bar{H}_{\alpha}^{+}(K) \leq \frac{(1+2 \alpha)^{d-1}(1+3 \alpha)-2}{2 \alpha^{d}}
$$

and

$$
\bar{H}_{\infty}^{+}(K) \leq 3 \cdot 2^{d-2} .
$$

Moreover, $\bar{H}_{\infty}^{+}(K)=3 \cdot 2^{d-2}$ if, and only if, $K$ is an affine $d$-cube.

Theorem 3. The cardinality of a family of pairwise touching positive homothetic copies of an o-symmetric convex body in $\mathbb{R}^{d}$ is strictly less than $3 \cdot 2^{d-1}$.

\section{Proof of Theorem 1}

We need the following, rather technical, definition and a lemma. 


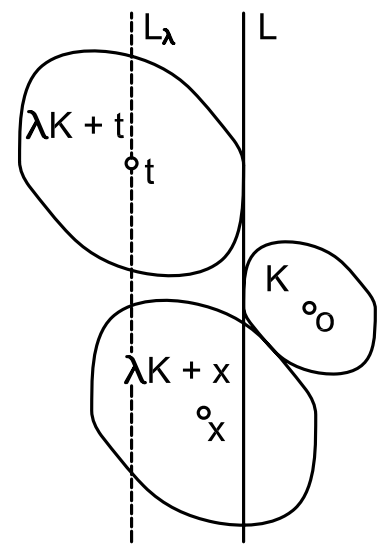

FIGURE 1 .

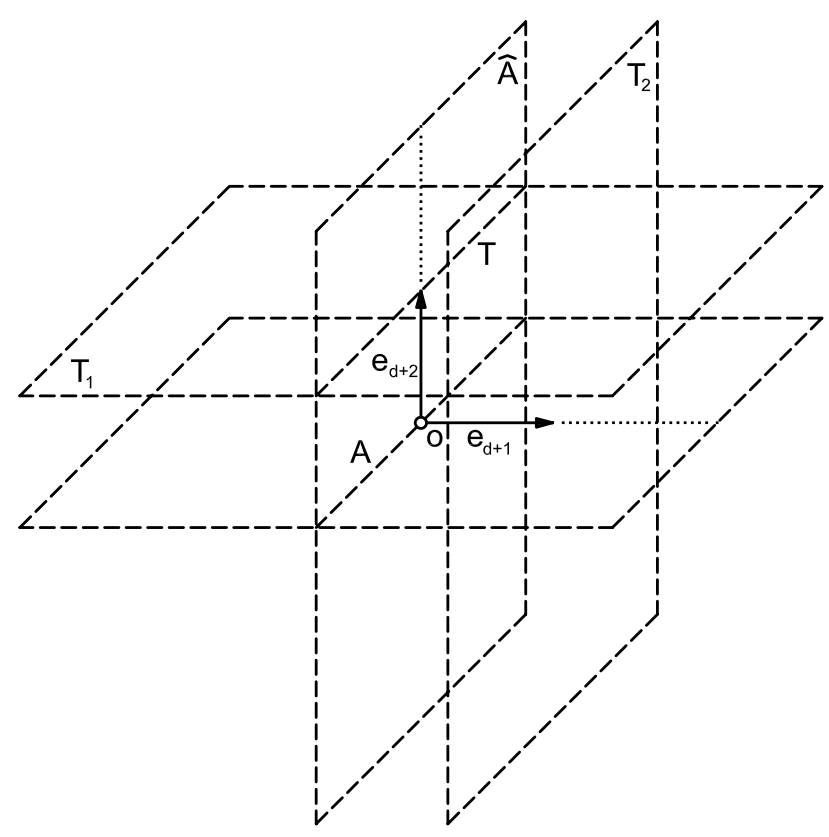

FIGURE 2.

Definition 4. Let $K \subset \mathbb{R}^{d}$ be a convex body that contains $o$ in its interior, and let $\lambda>0$. Furthermore, let $L$ be a hyperplane of $\mathbb{R}^{d}$ and $L^{+}$be one of the two closed half-spaces of $\mathbb{R}^{d}$ bounded by $L$. Then we call the set

$$
L_{\lambda}:=\left\{t \in L^{+}: \lambda K+t \text { touches } L\right\}
$$

the $\lambda K$-translate of $L$ into $L^{+}$.

We note that $L_{\lambda}$ is a translate of $L$.

Lemma 1. Let $K \subset \mathbb{R}^{d}$ be an o-symmetric convex body, and $\lambda K+x$ be a positive homothet of $K$ touching $K$. Let $L$ be a supporting hyperplane of $K$, and $L_{\lambda}$ be the $\lambda K$-translate of $L$ into the half-space that does not contain $K$.

Then $x$ is in the closed half-space that is bounded by $L_{\lambda}$ and contains $K$ (cf. Figure 1).

Proof. Suppose that $x$ is not in the closed half-space that is bounded by $L_{\lambda}$ and contains $K$. Then, by the definition of $L_{\lambda}$, we have that $\lambda K+x$ is contained in the open half-space that is bounded by $L$ and does not contain $K$. Hence, $\lambda K+x$ does not touch $\lambda K+x$, a contradiction.

Proof of Theorem 1. Let $e_{1}, \ldots, e_{d+2}$ be the standard basis of $\mathbb{R}^{d+2}$. To prove the equivalence of (1) and (2), we regard $\mathbb{R}^{d+1}$ and $\mathbb{R}^{d}$ as the affine subspaces $\hat{A}:=$ $\left\{x \in \mathbb{R}^{d+2}:\left\langle x, e_{d+1}\right\rangle=0\right\}$ and $A:=\left\{x \in \mathbb{R}^{d+2}:\left\langle x, e_{i}\right\rangle=0\right.$ for $\left.i=d+1, d+2\right\}$ of 
$\mathbb{R}^{d+2}$, respectively. We need also the following affine subspaces:

$$
\begin{aligned}
T_{1} & :=\left\{v \in \mathbb{R}^{d+2}:\left\langle v, e_{d+2}\right\rangle=1\right\} \\
T_{2} & :=\left\{v \in \mathbb{R}^{d+2}:\left\langle v, e_{d+1}\right\rangle=1\right\} \\
T & :=\left\{v \in \mathbb{R}^{d+2}:\left\langle v, e_{d+2}\right\rangle=1 \text { and }\left\langle v, e_{d+1}\right\rangle=0\right\} .
\end{aligned}
$$

Note that $T_{1}$ and $T_{2}$ are hyperplanes in $\mathbb{R}^{d+2}, T$ is a $d$-flat and $T \subset T_{1}$. (cf. Figure 2).

First, we prove that (1) implies (2). Consider a family $\left\{\lambda_{i} K+x_{i}: i=1,2, \ldots, n\right\}$ of pairwise touching homothets of the $o$-symmetric $d$-dimensional convex body $K$ in $\mathbb{R}^{d}$. We let $K_{i}:=\lambda_{i} K+x_{i}+e_{d+2}$ and note that $\left\{K_{i}: i=1,2, \ldots, n\right\}$ is also a family of pairwise touching homothets of $K$, which is contained in $T$. We set

$$
X^{\prime}:=\left\{x_{i}^{\prime}:=x_{i}+\lambda_{i} e_{d+1}+e_{d+2} \in \mathbb{R}^{d+2}: i=1,2, \ldots, n\right\} .
$$

Observe that $X^{\prime} \subset T_{1}$.

For any pair of distinct indices $i, j$, let $L(i, j)$ be an affine $(d-1)$-flat which separates $K_{i}$ and $K_{j}$ in $T$. Furthermore, let $H_{k}(i, j):=\operatorname{aff}\left(L(i, j) \cup\left\{x_{k}^{\prime}\right\}\right)$ for $k \in\{i, j\}$. Observe that $H_{i}(i, j), H_{j}(i, j) \subset T_{1}$, and they partition $T_{1}$ into four closed convex cones, exactly one of which contains both $x_{i}^{\prime}$ and $x_{j}^{\prime}$. We denote this cone by $C(i, j)$.

By Lemma 1 and the construction of $X^{\prime}$, we have that $X^{\prime}$ is a subset of $C(i, j)$. Thus, we have shown the following property of $X^{\prime}$.

(*): For every pair of distinct indices $i, j$, there is a cone $C(i, j)$ in $T_{1}$ containing $X^{\prime}$. The cone $C(i, j)$ is the intersection of two closed half-spaces $Z_{i}$ and $Z_{j}$ of $T_{1}$, such that $x_{i}^{\prime} \in \operatorname{relbd} Z_{i}, x_{j}^{\prime} \in \operatorname{relbd} Z_{j}$, and $L(i, j):=\left(\operatorname{relbd} Z_{i}\right) \cap$ $\left(\right.$ relbd $\left.Z_{j}\right)$ is an affine subspace of $T$ with $\operatorname{dim} L(i, j)=d-1$. Furthermore, $C(i, j)$ is symmetric about any line of the form $\left\{z+t e_{d+1}: t \in \mathbb{R}\right\}$, where $z \in L(i, j)$.

Let $X^{\prime \prime}$ denote the image of $X^{\prime}$ under the central projection from the origin $o$ onto the hyperplane $T_{2}$ of $\mathbb{R}^{d+2}$. More specifically, let $X^{\prime \prime}:=\left\{\frac{1}{\lambda_{i}} x_{i}+e_{d+1}+\frac{1}{\lambda_{i}} e_{d+2}: i=\right.$ $1, \ldots, n\}$. Note that $X^{\prime \prime}$ is contained in the open half-space $\left\{v \in T_{2}:\left\langle v, e_{d+2}\right\rangle>0\right\}$ of $T_{2}$. Consider the image $S$ of $X^{\prime \prime}$ under the orthogonal projection of $T_{2}$ onto the hyperplane $\hat{A}=\mathbb{R}^{d+1}$. From (*) and the previous remark, $S$ is a symmetrically antipodal set in $\mathbb{R}^{d+1}$ such that $o \notin \operatorname{conv} S$.

Now we prove that (2) implies (1). Let $S \subset \mathbb{R}^{d+1}$ be a symmetrically antipodal set of $n$ points such that $o \notin \operatorname{conv} S$. Reversing our consideration in the previous part, we may clearly construct a set $X^{\prime}=\left\{x_{i}^{\prime}: i=1,2, \ldots n\right\}$ such that $X^{\prime} \subset T_{1}$, $\lambda_{i}:=\left\langle x_{i}^{\prime}, e_{d+1}\right\rangle>0$, and $X^{\prime}$ satisfies $\left(^{*}\right)$.

Let $x_{i}:=x_{i}^{\prime}-\lambda_{i} e_{d+1}$ for $i=1,2, \ldots, n$. Clearly, $x_{i} \in T$. Set

$$
K:=\operatorname{conv}\left\{\frac{x_{i}-x_{j}}{\lambda_{i}+\lambda_{j}}: i, j=1, \ldots, n\right\}
$$

and note that $K$ is an $o$-symmetric compact, convex set in $A=\mathbb{R}^{d}$.

Assume that $\operatorname{dim} K=d$. We want to show that $\left\{\lambda_{i} K+x_{i}-e_{d+2}: i=1,2, \ldots, n\right\}$ is a family of pairwise touching positive homothets of the convex body $K$ in $A=\mathbb{R}^{d}$. 
Clearly, it is sufficient to show that $K_{i}:=\lambda_{i} K+x_{i}$ and $K_{j}:=\lambda_{j} K+x_{j}$ touch in $T$ for every $i \neq j$.

Since $x_{i}+\frac{\lambda_{i}}{\lambda_{i}+\lambda_{j}}\left(x_{j}-x_{i}\right)=x_{j}+\frac{\lambda_{j}}{\lambda_{i}+\lambda_{j}}\left(x_{i}-x_{j}\right)$, we obtain that $K_{i}$ and $K_{j}$ intersect. It remains to show that, say, $K_{1}$ and $K_{2}$ are separated by a $(d-1)$ dimensional affine subspace in $T$. Then

Let $L:=L(1,2)$ be the $(d-1)$-dimensional affine subspace in $T$ described in $\left(^{*}\right)$.

$$
L=\{x \in T:\langle x, u\rangle=0\}=\left\{x \in T_{1}:\langle x, u\rangle=\left\langle x, e_{d+1}\right\rangle=0\right\}
$$

for some vector $u \in A=\mathbb{R}^{d}$. We may assume that, say, $\left\langle u, x_{1}\right\rangle>0$. Then the symmetry of the cone $C:=C(1,2)$ in $(*)$ yields that $\left\langle u, x_{2}\right\rangle<0$.

Let $u_{i}:=u-\frac{\left\langle u, x_{i}\right\rangle}{\lambda_{i}} e_{d+1}$ for $i=1,2$. It is simple to check that $\left\langle u_{i}, x\right\rangle=0$ for any $x \in L$, and $\left\langle u_{i}, x_{i}^{\prime}\right\rangle=0$. An easy computation yields that $\left\langle u_{1}, x_{2}^{\prime}\right\rangle<0$ and $\left\langle u_{2}, x_{1}^{\prime}\right\rangle>0$, and thus

$$
C=\left\{x \in T_{1}:\left\langle u_{1}, x\right\rangle \leq 0 \text { and }\left\langle u_{2}, x\right\rangle \geq 0\right\} .
$$

Observe that the symmetry of the cone implies that $\left\langle u, x_{2}\right\rangle / \lambda_{2}=-\left\langle u, x_{1}\right\rangle / \lambda_{1}$.

From (*) it follows that $\left\langle u_{1}, x_{k}^{\prime}\right\rangle \leq 0$ and $\left\langle u_{2}, x_{k}^{\prime}\right\rangle \geq 0$ for $k=1,2, \ldots, n$. After substituting the definitions of $u_{1}$ and $u_{2}$, and $x_{k}^{\prime}=x_{k}+\lambda_{k} e_{d+1}$, we have that

$$
-\frac{\left\langle u, x_{1}\right\rangle}{\lambda_{1}} \leq \frac{\left\langle u, x_{k}\right\rangle}{\lambda_{k}} \leq \frac{\left\langle u, x_{1}\right\rangle}{\lambda_{1}} .
$$

We show that $L$ separates $K_{1}$ and $K_{2}$. By symmetry, it is sufficient to show that $\langle u, x\rangle \geq 0$ for any $x \in K_{1}$. By (5), for every $j, k$ we have

$$
\begin{gathered}
\left\langle u, x_{1}+\frac{\lambda_{1}}{\lambda_{j}+\lambda_{k}}\left(x_{j}-x_{k}\right)\right\rangle=\left\langle u, x_{1}\right\rangle+\frac{\lambda_{1}}{\lambda_{j}+\lambda_{k}}\left(\left\langle u, x_{j}\right\rangle-\left\langle u, x_{k}\right\rangle\right) \geq \\
\left\langle u, x_{1}\right\rangle+\frac{\lambda_{1}}{\lambda_{j}+\lambda_{k}}\left(-\frac{\lambda_{j}}{\lambda_{1}}\left\langle u, x_{1}\right\rangle-\frac{\lambda_{k}}{\lambda_{1}}\left\langle u, x_{1}\right\rangle\right)=0,
\end{gathered}
$$

which yields our statement if $\operatorname{dim} K=d$. If $\operatorname{dim} K<d$, a similar argument may be applied.

Now we show that (2) implies (3). Let $S \subset \mathbb{R}^{d+1}$ be a symmetrically antipodal set such that $o \notin \operatorname{conv} S$. For any pair of distinct points $p, q \in S$, let $H_{p, q}$ denote a hyperplane such that $p \in H_{p, q}, q \in-H_{p, q}$, and both $H_{p, q}$ and $-H_{p, q}$ support conv $S$. Let $S_{p, q}$ be the strip bounded by $H_{p, q}$ and $-H_{p, q}$, and $C$ be an $o$-symmetric cube with $S \subset C$. Set

$$
K:=C \cap\left(\bigcap_{\substack{p, q \in S \\ p \neq q}} S_{p, q}\right) .
$$

Note that $K$ is an $o$-symmetric convex body in $\mathbb{R}^{d+1}, S$ is a diametral set in $K$, and $o \notin \operatorname{conv} S$. Thus (2) yields (3). Since (3) clearly implies (2), we have that (2) and (3) are equivalent.

The equivalence of (3) and (4) is easy to show, hence we omit it. Observe that $S$ in (3) corresponds to the set of translation vectors in (4). 


\section{Proof of Theorem 2}

Our proof is based on the idea of the proof of the Theorem of [1]. We begin with a variant of the Lemma of [1].

Lemma 2. Let $f$ be a function on $[0,1]$ with the properties $f(0) \geq 0, f$ is positive and monotone increasing on $(0,1]$, and $f(x)=(g(x))^{k}$ for some concave function $g$ with $k>0$. Then

$$
F(x):=\frac{1}{f(x)} \int_{0}^{x} f(z) \mathrm{d} z
$$

is strictly increasing on $(0,1]$.

Proof of Lemma 2. Let $0<x<y \leq 1$. We want to show that $F(x)<F(y)$ or, equivalently, that

$$
\frac{f(y)-f(x)}{f(x)} \int_{0}^{x} f(z) \mathrm{d} z<\int_{x}^{y} f(z) \mathrm{d} z .
$$

This inequality trivially holds if $f(x)=f(y)$, and thus we may assume that $f(x)<f(y)$. Let $L(z):=\frac{g(y)-g(x)}{y-x}(z-x)+g(x)$ for $z \in[0, y]$. Note that since $g(z)$ is concave and $k>0$, we have $f(z) \geq(L(z))^{k}$ for every $z \in[x, y]$, and $f(z) \leq(L(z))^{k}$ for every $z \in(0, x]$. Thus, it is sufficient to prove that

$$
T:=\int_{x}^{y}(L(z))^{k} \mathrm{~d} z-\frac{(g(y))^{k}-(g(x))^{k}}{(g(x))^{k}} \int_{0}^{x}(L(z))^{k} \mathrm{~d} z>0 .
$$

After integrating and simplifying, we obtain that

$$
T=\frac{(g(y))^{k}(y-x)}{k+1}+\frac{\left((g(y))^{k}-(g(x))^{k}\right)(y g(x)-x g(y))^{k+1}}{(g(x))^{k}(k+1)(g(y)-g(x))(y-x)^{k}} .
$$

Note that from $0<f(z), z \in(0,1]$, we have that $L(0) \geq 0$, which yields that $0<\frac{g(y)}{y} \leq \frac{g(x)}{x}$. Thus, the second member of $T$ is nonnegative, and the inequality in (6) immediately follows.

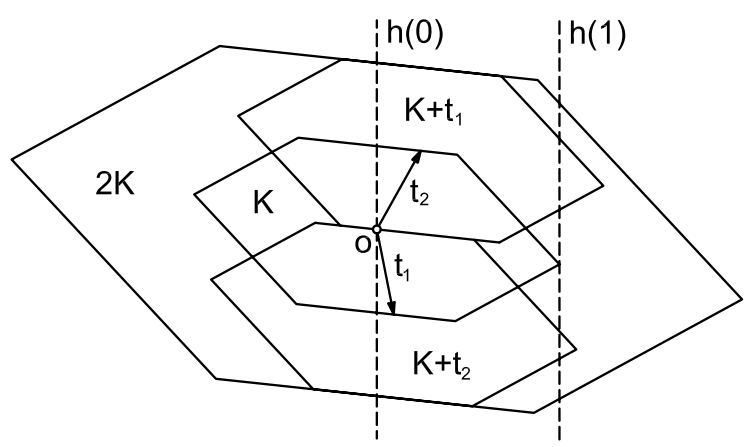

Figure 3 
Proof of Theorem 2. First, we present a detailed proof of (2) and discuss the case of equality. Next, we outline the proof of (1), which is essentially the same as that of (2).

Let $v \in \mathbb{S}^{d-1}$ and let $K+t_{1}, K+t_{2}, \ldots, K+t_{n}$ be pairwise non-overlapping translates of $K$ such that $o \in K+t_{i}$ and $a_{i}:=\left\langle t_{i}, v\right\rangle \geq 0$ for $i=1,2, \ldots, n$. Set $h(x):=\left\{p \in \mathbb{R}^{d}:\langle p, v\rangle=x\right\}$. Without loss of generality, we may assume that $h(-1)$ and $h(1)$ are supporting hyperplanes of $K$. Note that $K+t_{i}$ is between $h(-1)$ and $h(2)$, and it is contained also in $2 K$, for $i=1, \ldots, n$ (cf. Figure 3). Let $\mathrm{V}_{d}($.$\left.) (resp. \mathrm{V}_{d-1}().\right)$ denote $d$-dimensional (resp. $(d-1)$-dimensional) volume. Then

$$
\int_{-1}^{2} \mathrm{~V}_{d-1}\left(\left(\bigcup_{i=1}^{n} K+t_{i}\right) \cap h(x)\right) \mathrm{d} x=n \mathrm{~V}_{d}(K) .
$$

We separate the above integral into two parts and estimate them separately. First, note that

$$
\int_{0}^{2} \mathrm{~V}_{d-1}\left(\left(\bigcup_{i=1}^{n} K+t_{i}\right) \cap h(x)\right) \mathrm{d} x \leq \int_{0}^{2} \mathrm{~V}_{d-1}(2 K \cap h(x)) \mathrm{d} x=2^{d-1} \mathrm{~V}_{d}(K)
$$

Set $f(x):=\mathrm{V}_{d-1}(K \cap h(x-1))$, and observe that $f(0) \geq 0, f$ is positive and monotone increasing on $(0,1]$, and $f(.)^{\frac{1}{d-1}}$ is concave by the Brunn-Minkowski inequality. By Lemma 2,

$$
\begin{aligned}
& \int_{-1}^{0} \mathrm{~V}_{d-1}\left(\left(\bigcup_{i=1}^{n}\left(K+t_{i}\right)\right) \cap h(x)\right) \mathrm{d} x=\sum_{i=1}^{n} \int_{0}^{1} \mathrm{~V}_{d-1}\left(K \cap\left(h(x-1)-t_{i}\right)\right) \mathrm{d} x \\
= & \sum_{i=1}^{n} \int_{0}^{1-a_{i}} f(x) \mathrm{d} x \leq \sum_{i=1}^{n} \int_{0}^{1} f(x) \mathrm{d} x \frac{f\left(1-a_{i}\right)}{f(1)}=\frac{\mathrm{V}_{d}(K)}{2 f(1)} \sum_{i=1}^{n} \mathrm{~V}_{d-1}\left(K \cap h\left(-a_{i}\right)\right) \\
= & \frac{\mathrm{V}_{d}(K)}{2 f(1)} \mathrm{V}_{d-1}\left(\left(\bigcup_{i=1}^{n}\left(K+t_{i}\right)\right) \cap h(0)\right) \leq \frac{\mathrm{V}_{d}(K)}{2 f(1)} \mathrm{V}_{d-1}(2 K \cap h(0)) \\
= & \frac{1}{2} \mathrm{~V}_{d}(K) \frac{\mathrm{V}_{d-1}(2 K \cap h(0))}{\mathrm{V}_{d-1}(K \cap h(0))}=2^{d-2} \mathrm{~V}_{d}(K) .
\end{aligned}
$$

This inequality, combined with (7) and (8), yields (2).

Now we prove the equality part of the theorem. Assume that $n=3 \cdot 2^{d-2}$. Then, by Lemma 2, we have two possibilities. One is that $f(0)=0$, and $a_{i}=0$ or $a_{i}=1$ for each $i=1, \ldots, n$. The other one is that $f(0)>0$ and $a_{i}=0$ for each $i=1, \ldots, n$.

If $f(0)>0$ then $a_{i}=0$ for each $i=1, \ldots, n$, and hence, $\left\{\left(K+t_{i}\right) \cap h(0): i=\right.$ $1,2, \ldots, n\}$ is a family of pairwise non-overlapping translates of $K \cap h(0)$ in $h(0)$, each of which contains $o$. Thus, [8] implies that $n \leq 2^{d-1}$, which contradicts our assumption.

Assume that $f(0)=0$, and $a_{i}=0$ or $a_{i}=1$ for $i=1, \ldots, n$. Observe that the family $\left\{\left(K+t_{i}\right) \cap h(0): a_{i}=0\right\}$ tiles $2 K \cap h(0)$, hence $a_{i}=0$ for $2^{d-1}$ values 
of $i$, and $K \cap h(0)$ is an affine $(d-1)$-cube. There is only the obvious way such that translates of $K \cap h(0)$ tile $2 K \cap h(0)$, and so we obtain that $\left\{t_{i}: a_{i}=0\right\}$ is an $o$-symmetric set. Moreover, $\left\{K \pm t_{i}: i=1,2, \ldots, n\right\}$ is a family of pairwise non-overlapping translates of $K$, all containing the origin. By [8], the cardinality of this family is at most $2^{d}$, with equality if, and only if, $K$ is an affine $d$-cube. On the other hand, since $n=3 \cdot 2^{d-2}$, we obtain that card $\left\{i: a_{i}=1\right\}=2^{d-2}$. Hence $\operatorname{card}\left\{ \pm t_{i}: i=1, \ldots, n\right\}=2^{d-1}+2 \cdot 2^{d-2}=2^{d}$, and $K$ is an affine $d$-cube.

Next, let $K:=[-1,1]^{d}$. Note that there are $3 \cdot 2^{d-2}$ vectors $\left(v_{1}, v_{2}, \ldots, v_{n}\right) \in$ $\{-1,1\}^{d}$, whose coordinates satisfy the inequality $v_{1}-v_{2} \geq 0$. The translates of $K$ by these vectors are pairwise non-overlapping, and each of them contains the origin. Thus, (2) is sharp for the cube.

Finally, we outline the proof of (1). Let $\alpha K+t_{1}, \alpha K+t_{2}, \ldots, \alpha K+t_{n}$ be pairwise non-overlapping translates of $K$ that touch $K$. Let $v, t_{i}, a_{i}, h(x)$ be as above. Note that $\alpha K+t_{i}$ is between $h(-\alpha)$ and $h(1+2 \alpha)$, and it is contained also in $(1+2 \alpha) K$, for $i=1, \ldots, n$. Thus,

$$
\int_{-\alpha}^{1+2 \alpha} \mathrm{V}_{d-1}\left(\left(\bigcup_{i=1}^{n} \alpha K+t_{i}\right) \cap h(x)\right) \mathrm{d} x=n \alpha^{d} \mathrm{~V}_{d}(K) \quad \text { and }
$$

$$
\int_{0}^{1+2 \alpha} \mathrm{V}_{d-1}\left(\left(\bigcup_{i=1}^{n} \alpha K+t_{i}\right) \cap h(x)\right) \mathrm{d} x
$$

$\leq \int_{0}^{1+2 \alpha} \mathrm{V}_{d-1}((1+2 \alpha) K \cap h(x)) \mathrm{d} x-\int_{0}^{1} \mathrm{~V}_{d-1}(K \cap h(x)) \mathrm{d} x=\frac{(1+2 \alpha)^{d}-1}{2} \mathrm{~V}_{d}(K)$.

Set $f(x):=\mathrm{V}_{d-1}(\alpha K \cap h(x-\alpha))$, and observe that the conditions of Lemma 2 are satisfied by $f$. We may assume that $a_{1}, \ldots, a_{m} \leq \alpha<a_{m+1}, \ldots, a_{n}$. By Lemma 2,

$$
\begin{aligned}
& \int_{-\alpha}^{0} \mathrm{~V}_{d-1}\left(\left(\bigcup_{i=1}^{n}\left(\alpha K+t_{i}\right)\right) \cap h(x)\right) \mathrm{d} x=\sum_{i=1}^{m} \int_{0}^{\alpha-a_{i}} f(x) \mathrm{d} x \leq \sum_{i=1}^{m} \int_{0}^{\alpha} f(x) \mathrm{d} x \frac{f\left(\alpha-a_{i}\right)}{f(\alpha)} \\
= & \frac{\alpha^{d} \mathrm{~V}_{d}(K)}{2 f(\alpha)} \sum_{i=1}^{m} \mathrm{~V}_{d-1}\left(\left(\alpha K+t_{i}\right) \cap h(0)\right)=\frac{\alpha^{d} \mathrm{~V}_{d}(K)}{2 f(\alpha)} \mathrm{V}_{d-1}\left(\left(\bigcup_{i=1}^{m}\left(\alpha K+t_{i}\right)\right) \cap h(0)\right) \\
\leq & \frac{\alpha^{d} \mathrm{~V}_{d}(K)}{2 f(\alpha)}\left[\mathrm{V}_{d-1}((1+2 \alpha) K \cap h(0))-\mathrm{V}_{d-1}(K \cap h(0))\right]=\frac{\alpha(1+2 \alpha)^{d-1}-1}{2} \mathrm{~V}_{d}(K) .
\end{aligned}
$$

This inequality, combined with (9) and (10), yields (1).

\section{REFERENCES}

[1] Károly Bezdek and Peter Brass, On $k^{+}{ }_{-}$neighbour packings and one-sided Hadwiger configurations, Beiträge Algebra Geom. 44 (2003), no. 2, 493-498. MR 2004i:52017

[2] Károly Bezdek and Robert Connelly, Intersection points, Ann. Univ. Sci. Budapest. Eötvös Sect. Math. 31 (1988), 115-127 (1989). MR 90i:52014 
[3] Károly Böröczky, Jr., Finite Packing and Covering, Cambridge Tracts in Mathematics, vol. 154, Cambridge University Press, Cambridge, 2004. MR 2005g:52045

[4] L. Danzer and B. Grünbaum, Über zwei Probleme bezüglich konvexer Körper von P. Erdös und von V. L. Klee, Math. Z. 79 (1962), 95-99. MR 25 \#1488

[5] L. Fejes Tóth, Über eine affininvariante Maßzahl bei Eipolyedern, Studia Sci. Math. Hungar. 5 (1970), 173-180. MR 42 \#3673

[6] H. Hadwiger, Über Treffanzahlen bei translationsgleichen Eikörpern, Arch. Math. 8 (1957), 212-213. MR 19,977e

[7] Márton Naszódi, On a conjecture of Károly Bezdek and János Pach, Period. Math. Hungar. 53 (2006), no. 1-2, 227-230. MR 2286473

[8] C. M. Petty, Equilateral sets in Minkowski spaces, Proc. Amer. Math. Soc. 29 (1971), 369-374. MR 43 \#1051

Zsolt Lángi, Dept. of Geometry, Budapest University of Technology, Budapest, Egry József u. 1., Hungary, 1111

E-mail address: zlangi@math.bme.hu

Márton Naszódi, Dept. of Math. and Stats., University of Alberta, 632 Central Academic Building, Edmonton, Ab, Canada T6G 2G1

E-mail address: mnaszodi@math.ualberta.ca 